\title{
Adaptability and Flexibility of the Human Motor System: Implications for Neurological Rehabilitation
}

\author{
Theo Mulder ${ }^{1}$ and Jacqueline Hochstenbach ${ }^{2,3}$ \\ ${ }^{1}$ Institute of Human Movement Sciences and ${ }^{2}$ Department of Rehabilitation Medicine, \\ ${ }^{3}$ Neuropsychological Center for Brain Damage Aftercare, University of Groningen, \\ Groningen, The Netherlands
}

\begin{abstract}
This article stresses the plasticity of the adult sensorimotor cortex in response to various injuries or environmental changes. The dominant role of sensory input is discussed. A number of studies are presented that show how input may lead to learning and change. Learning is discussed in relation to recovery. It is shown how concepts from the field of motor control and learning may be used for improving neurological rehabilitation. Specific attention is given to the variability of input, the meaningfulness of input, and the role of the learning context. The learning context and the application context should have essential characteristics in common, otherwise transfer of learning will be non-optimal. It is argued that learning landscapes are necessary in order to treat patients in such a way that the learned skills are transferable to situations outside the hospital.
\end{abstract}

\section{KEY WORDS}

recovery, neurological rehabilitation, motor disorders, brain damage

\section{INTRODUCTION}

Each year hundreds of thousands of people are hit by brain damage. Most of them suffer from the consequences of a stroke. In general, one can argue that about 500 cases occur annually in a population of 250,000 (Dombovy, 1993). Stroke is the third commonest cause of death after heart disease and cancer in the western society and the most frequent cause of severe disability (Khaw, 1996). Although stroke is seen as a disease of old age, with age being the most important risk factor, approximately $25 \%$ of all stroke victims are younger than 65 years and thus disabled during the productive years of their lives.

Although primary prevention still seems to be the most important means of reducing the extensive effects of stroke, it is not applicable to all risk factors. In recent years, research has been increasingly devoted to the effects of restorative pharmacological interventions and neuroprotectives immediately following infarction, but until now the results are ambiguous (James, 1997). An increasing number of researchers even wonder whether the possibility of neuroprotection is a valid concept in humans and whether further research is still sensible (Lodder, in press). This means that after surviving the acute phase, most patients and their families still are confronted with a complex mixture of sensorimotor, cognitive, and behavioral problems (Hochstenbach et al., 1998). These patients are referred to a rehabilitation center, be it that the percentage of referrals may differ substantially across different countries. In the Netherlands, about $5 \%$ to $10 \%$ of all stroke patients are referred to a rehabilitation center. 
Although many agree upon the usefulness of rehabilitation, it is very difficult to assess the efficacy of neurological rehabilitation. Many variables influence the end result, and most of the studies differ in methodology so that comparability is limited (see also Wagenaar, 1990, Kwakkel, 1998). In this article, we will not focus on the efficacy of neurological rehabilitation in terms of an analysis of costs and benefits but ask whether neurological rehabilitation may be improved by linking it more closely to modern developments in neurobiology, movement science, and neuropsychology. Indeed, in spite of the massive amounts of time and money that are invested in neurological rehabilitation, the results are not always convincing. To answer the above mentioned question, we will describe in gross terms some recent insights into the intrinsic plasticity of the neuromotor system. We argue that these insights are relevant for the (re-)learning of motor skills after brain damage.

\section{THE INTRINSIC PLASTICITY OF THE NEUROMOTOR SYSTEM}

The largest part of the $20^{\text {th }}$ century was dominated by a rather pessimistic view on the plasticity of the brain. It was argued that a hardwired brain with localization of function left no room for plasticity (see Bach-y-Rita, in press). Furthermore, it was argued that the adult brain had no intrinsic capacity to recover from damage. The focus in treatment, therefore, was very often on functional compensation.

During the eighties of the last century, however, a shift in thinking occurred, mainly as a result of the studies performed by Merzenich and co-workers. Merzenich et al. (see Merzenich \& Kaas, 1983) were able to show that neural maps on the sensorimotor cortex were not rigid but very dynamic. The maps changed under the influence of input. When input was withdrawn, the maps more or less shriveled up, whereas when input was increased, the maps extended in space. Furthermore, they showed that previously existing synapses could be dramatically modified and that new synapses could be formed. Many other studies showed that central sensory representations could be reorganized, not only as a result of changes in the peripheral input in an experimental context (Kaas et al., 1983) but also after amputation (Hall, et al., 1990), spinal cord injury (Topka et al., 1991; Bruehlmeier et al., 1998), deafferentation (Brasil-Neto et al., 1992), after ischaemic nerve block (Brasil-Neto et al., 1993), and after stroke (Traversa et al., 1997).

In earlier work, Spear (1997) showed that neurons in the lateral supra-sylvian gyrus that normally are unresponsive to specific visual input became responsive to these stimuli when the visual cortical areas were impaired. Cohen et al. (1999) showed the existence of cross-modal plasticity in the blind. Areas activated in sighted subjects during the performance of visual tasks became activated in blind subjects in association with tactile or auditory discrimination tasks. They indicated, however, that the susceptible period for this form of functionally relevant cross-modal plasticity did not extend beyond the age of 14 years. Calford \& Tweedale (1990) mentioned that the adaptive plasticity even showed inter-hemispheric transfer. They showed that in flying foxes (Pteropus scapulatus) the receptive fields of neuron assemblies, which originally represented the thumb that had been anaesthetized, expanded within a few minutes after the injection. The receptive fields in the opposite hemisphere representing the unaffected thumb, however, also expanded.

These results clearly emphasized the plasticity of the system, and Nudo et al. (1996) indicated that this plasticity could be exploited for rehabilitative training. After an ischemic infarct that destructed the hand-territory in the primary motor cortex, squirrel monkeys were trained in skilled hand-use. Nudo et al. were able to obtain evidence for a 
functional reorganization. In some instances, the hand representation expanded into regions formerly occupied by representations of the elbow and shoulder. More important was that this functional reorganization was accompanied by a behavioral recovery of skilled hand function. Their results suggested that after local damage to the motor cortex, rehabilitative training may facilitate reorganization and that the undamaged motor cortex plays a role in recovery. In other words, the motor cortex reorganizes in association with the acquisition of skills.

Recently, Stefan et al. (2000) showed that an enduring change in the excitability of cortical output circuits could be induced by synchronously pairing low-frequency peripheral stimulation of somatosensory afferents with transcranial magnetic stimulation over the motor cortex. They argued that this paired associative stimulation activated intracortical fibers. Remarkably few stimulus pairs were necessary to induce this change in cortical excitability. The plastic effect was already present after 30 minutes, or 90 stimulus pairs. The effect lasted between 30 and 60 minutes, which refers to a Long Term Potential-like (LTP) phenomenon, as LTP by definition lasts $>30$ minutes (see, Stefan et al., 2000).

Gaining more insight into the mechanisms behind cortical plasticity is extremely important and a necessary requirement for the development of novel treatment and training procedures.

The above mentioned examples clearly indicate that sensory input is critical for the maintenance of stable input-output relations. Absence of any input or a degraded input from a limb has an almost immediate effect on the cortical representation of that limb. Against this background Rosinni et al., $(1994,176)$ argued that, "continuous peripheral input from the whole of a given body district is a conditio sine qua non for the maintenance of normal somatotopic cortical organization related to that body part." Ramachandran \& Hirstein (1998) showed what happened when afferent input to the brain was shut off completely. After amputation of a limb, patients experience very vivid phantom sensations, that is, they still experience the presence of a leg or hand, in spite of the fact that the limb has been removed. These patients report bizarre changes across time, such as telescoping or shrinkage of the phantom limb so that, for example their amputated foot finally is felt directly connected to the knee or hip. Also, the overall size of the limb may shrink, even to the size of a stamp. Their results also indicated that the removal of a limb resulted in a number of remarkable reorganizations. For example, when the investigator stroked the face of a patient, the patient experienced a similar sensation in his phantom hand. Water that runs down the face was experienced as water not only running down the face but also running down the amputated hand. The authors explained this phenomenon in terms of the re-mapping of brain areas. Indeed, in the Penfield 'homunculus' (the representation of body parts onto the surface of the brain) it can be observed how the hand area is bordered below by the face area. Due to the amputation, input from the hand area was lost and consequently sensory fibers originating from the face invaded the 'empty' area of the hand and began to drive the cells there. As a result of this neural invasion, it became possible to 'touch' the phantom hand by touching the face. The same happened in the shoulder area that is represented just above the hand area in the Penfield 'homunculus', touching points on the shoulder evoked sensations in the phantom hand (see also Ramachandran \& Blakeslee, 1998; Spitzer, 1999).

All the above described examples stress the crucial role of information for the maintenance of sensory maps. As soon as the information is withdrawn, the maps decay. The effect of this decay is not limited solely to the sensory domain but has implications also for the motor domain. A large number of studies showed that animals who 
have been deafferented after having learned a skill continue to perform the skill, but with distinct performance limitations. They are less fast, less fluent, and less coordinated (see, Magill, 1998). Also for learning, sensory information is crucially needed. Numerous studies showed that in conditions where no information was fed back concerning the results of the performed movements, learning was absent or minimal (Schmidt, 1988).

\section{LEARNING}

Although learning forms a classic topic in psychology, it is still difficult to define unambiguously. Normally, learning is defined as a relatively permanent change in behavior as a result of practice (Schmidt, 1988; Magill, 1998). This definition focuses totally on the end-result of learning and ignores the process of learning. It is, however, very difficult to observe learning in vivo, since it must be shown that the cellular changes are specifically related to the effects of experience and not to some other property of the milieu or to spontaneous development. Although learning depends on changes in neurons and their circuitry, the basic mechanisms behind these changes are still far from understood. During the last decades, many experiments have been performed that have improved our insight into the basic characteristics of motor learning. These studies indicated that as a consequence of learning,

- new dendrites may sprout, or there might be extension of already existing branches;

- existing synapses may change or new ones created;

- changes in the axons may take place; or

- new neurochemicals may be produced.

In spite of the many obscurities that still exist, it is generally accepted that learning is based on the intensification of the connectivity between neurons. The co-activation of connected cells results in a modification of weights so that the probability increases that a post-synaptic cell will fire when a pre-synaptic cell fires. This, in fact, is the essence of Donald Hebb's insight, pictured in his highly influential book The Organization of Behavior (Hebb, 1949). It took, however, more than 20 years until physiological evidence for this epoch-making idea could be obtained.

In 1973, Bliss \& Lømo showed that when input fibers to the hippocampus were stimulated by brief high-frequency volleys, a long-lasting increase of the connection strength between the input fibers and their target neurons resulted. What Bliss \& Lømo discovered became known as long term potentiation (LTP). Their finding attracted considerable attention because it provided a model of how converging inputs from various sources, including local intracortical fibers, cortico-cortical and thalamo-cortical afferents could interact to reshape local patterns and representations. Their experimental results supported to a large extent the ideas of Hebb (see also Churchland \& Sejnowski, 1992).

In search for the basic mechanisms of learning, numerous experiments were performed on hippocampal neurons. However, these experiments were influenced also by some remarkable studies performed by neuropsychologists (see, Milner et al., 1968). These psychologists studied patients who underwent a bilateral resection of the mesial temporal lobe structures for the management of intractable epilepsy. It was found that after the operation, the patients were left almost totally amnesic. They could not remember events that had happened as recently as 5 minutes before, even when the events were salient and important to them. They had lost the ability to learn and retain novel information, although they were unimpaired in memorizing events that had taken place before the surgery. Warrington \& Weiskrantz (1974) reported the intriguing result that these patients 
learned tasks, such as the picture-completion task, at a normal rate, although they were amnesic and denied that they had ever seen the pictures they completed correctly. This indicated that some form of learning exists that is implicit and that takes place even when the learner has no awareness of the learned material.

The LTP-phenomenon was not limited to the hippocampus but was also shown in a number of brain areas, such as the auditory cortex (Cruikshank \& Weinberger, 1996), the somatosensory cortex (Crair \& Malenka, 1995), and the visual cortex (Hirsch \& Gilbert, 1993). Recently, LTP has been demonstrated in cortical areas, known for their capacity to reorganize themselves spontaneously when input is changed (Aroniadou-Anderjaska \& Keller, 1995).

Hence, as a result of input, synaptic weights change and this change is termed learning. Spitzer $(1999,51)$ in his well written monograph argues that learning takes place by gradually changing the synaptic weights and the connectivity between groups of neurons. This means that learning has to occur slowly because only small changes of the weights enable the networks to extract general features from the input and to distil rules. If synaptic weights were changed in large increments, the target would be missed. Large changes might lead to wild oscillations of the network around the desired output rather than slowly progressing towards a stable input-output relationship.

\section{MOTOR LEARNING AND RECOVERY}

Processes playing a role in recovery after damage to the neuro-motor system are partly identical to learning. The damage forces the system to reorganize and to create a novel order. A stroke, for example, results in a dramatic breakdown of many central control systems, leading to severe sensorimotor and cognitive disorders. A limb-amputation leaves the central machinery intact but alters the layout of the afferent input to the brain so that central systems have to adapt to this altered input. A progressive neuromuscular disease (e.g., Charcot Marie Tooth, type Ia) leads to changes in the afferent input to the brain, but as these changes emerge slowly across the years, they leave the brain ample time to adapt to these changes (see Mulder et al., in press). Hence, whether the damage is central or peripheral, whether the damage appears suddenly or as the result of a slowly progressing disease, in all these cases the neural system has to reorganize, has to change, has to re-learn. Learning, adaptation, and recovery, therefore, are related processes. Two types of learning may be distinguished: learning as a result of selforganization and learning as a result of exercise. The examples mentioned above all stress the selforganizing character of the system, in that the obtained changes took place as a result of response-produced input. Indeed, in all these experiments, there was no supervisor and no training protocol. When two fingers that are normally separated from each other are connected to each other by surgery, it can be observed that the separate representations of the individual fingers in the brain disappear, and the two fingers induce a single fusiform representation (see Allard et al., 1991). No exercise is needed for these changes, they take place as a result of the 'design' of the system. Information shapes the control.

However, not all changes take place in such an autonomous way, sometimes we have to train and exercise in order to reach our goals. The relearning of gait, reaching, or speech after brain damage clearly is the result of an interaction between the above described spontaneously occurring mechanisms and a carefully designed learning program. In the next part of the paper, we will focus on the designing of such a program, and we will argue that learning may be improved when recent insights of neuro-plasticity and motor learning are 
integrated with the methods of motor rehabilitation.

Four basic rules for motor (re-)learning are distinguished here (see also, Mulder \& Hochstenbach, in press):

1. input (information) is a sine qua non for learning;

2. input must be variable;

3. input must be meaningful,

4. the site of training must be related to the site of application (principle of equivalent situations).

\section{Input is a sine qua non for learning}

As has been described above, learning is the result of synaptic changes. These changes, however, take place only when the brain is fed with activitydependent information. Without any input or information the networks decay. Immobilization, therefore leads to the 'shrinkage' of neuronal networks. It is known that the continuous input of sensory feedback during gait does influence the strength of neuronal connections in the spine. In cats it has been shown that after spinal cord injury, step-like movements only reappear when afferent information is fed into the spinal cord (Wernig et al., 1998)

Also in spinal-cord injured humans it has been shown that some relearning of walking could be established as a result of intensive exercise on a treadmill (Dietz et al., 1998; see also, van de Crommert et al., 1998). Spinal cord circuits, as well as supraspinal centers ( in patients with incomplete lesions), are capable of significant reorganization as a result of activity-dependent input. Without this input, no reorganization takes place. The same is true for stroke patients; substantial improvement of gait is possible as a result of exercise and training. Each step, each new loco-motor task creates a flow of afferent input which forms the basis for learning.

\section{Input should be variable}

However, input as a repetitive train of identical sensory stimulation is not effective. The input should be variable. When identical input is repeated, the input looses its informational value and the system will quickly adapt to it. Neural networks are shaped by both input and variation. Note, however, that variation is not identical to chaos or random input. Indeed, the worst thing for a learning system is random input. If the input is random, no structure can be extracted from it and no learning takes place (Spitzer, 1999, but see also Ritter \& Schulten, 1986; Singer, 1986). The same, however, is true for identical inputs. When each movement produces identical information, the system is not able to distill knowledge (rules) from these movements, which can be transferred to other movements or to movements made in a novel context. The unique characteristic of movement, however, is that each response in fact is a novel one. That is, no response is produced in exactly the same way as before. Something about the response condition or context is always unique and leads to the immediate modification of the present response. In order to be able to cope with the instabilities in the environment, it is necessary to vary the conditions and performance-characteristics of the movements during learning or therapy. By varying the performance, also the performance-produced feedback (sensory input to the brain) is changing, which is important for shaping neural networks with a high generalization value. When a learning system is confronted with identical information across time, it will certainly learn from that information, but the learned skill will not be flexible and may not be generalized to other situations or movements that differ from the one learned on the basis of the available information.

The great Russian physiologist, Nicolai Bernstein, was well aware of these arguments when he stated 
that learning is repetition without repetition (see Whiting, 1984). What he meant was that repetition is necessary, but the repeated movements or actions should be different. Skill (re-)learning only results when the repeated input is variable. Also Spitzer (1999) stresses the importance of variable input, but he adds to it the need for small variations. When across attempts the input differs too much and too fast, the system will not be able to distill transferable information from these attempts.

The implications for retraining gait after stroke are clear: a patient should be exercised in a number of conditions which create changes in sensory input (e.g. walking on flat floors, sand, grass, gravel, uphill, down-hill). These conditions create different flows of input.

\section{Input should be meaningful}

Input should be variable, but it also should be meaningful. Merely presenting input patterns is not enough for adequate learning. The learner should be attentive to the offered information. The tasks employed in rehabilitation should, therefore, be significant for the patient. Without this significance the learning effect will be minimal. It is well known that information with an emotional implication is remembered better than neutral information (Le Doux, 1996). McGaugh et al., (1993) showed that if rats are given an injection of adrenaline, immediately after learning they show an enhanced memory for the learned task. This suggests that if adrenaline is released naturally from the adrenal glands, the learned task will be remembered better. Since emotional arousal usually results in the release of adrenaline, it might be expected that learning in situations with an emotional content or with a clear meaning for the person will be superior to other situations. McGaugh et al. (1990; cited in LeDoux, 1996, 207) gave additional evidence for the role adrenaline plays in the memory enhancing effect of emotional arousal. They asked adult subjects to read two versions of a story about a boy riding a bike. Some of the subjects where asked to read the version in which the boy takes a ride on his bike and goes home. $\mathrm{He}$ and his mom drive to the hospital to pick up his dad, a doctor. The other version was that the boy takes a ride on his bike, is hit by a car, and rushed to the hospital, where his dad is a doctor. The words in the two stories are matched as closely as possible, with only the emotional implications manipulated. After reading the story, and before being tested for recall, one group of subjects is injected with a placebo while the other group is injected with a drug that blocks the effect of adrenaline. For the placebo-treated subjects, those that read the emotional story remembered many more details than those that read the neutral story. However, for the subjects receiving the adrenaline blockade, there was no difference in the memory of the emotional and non-emotional stories. All subjects performed like the placebo group that read the neutral story. Adrenaline blockade indeed prevented the memory enhancing effects of emotional arousal.

Spitzer $(1999,144)$ addressed the importance of meaningful input by indicating that unimportant or meaningless events do not cause cortical changes, even if they occur with the same frequency as unimportant ones. When two groups of animals have to learn a certain discrimination task and only one group is rewarded for its attempts, then only this group shows a learning effect and a change of the cortical representation. So, training has an effect only when subjects pay attention to the stimuli, and subjects will pay attention to a task or stimulus when it has significance.

Corbetta et al. (1991) questioned whether attending to specific features of the environment has a measurable effect on brain functioning. Normal subjects watched a computer screen displaying up to thirty objects of a certain color and form, moving at a certain speed. The subjects had to attend to color or form or movement. 
Corbetta et al. recorded the brain activity by means of PET during rest, as well as when attending to the selected features. When we pay attention to specific aspects of the environment, the activity of the area that processes the selected information increases. The PET results indicated, that when subjects concentrated on color, form, or movement, the cortical areas that became active were identical with or close to the areas known to be involved in the processing of color, form or motion. Hence, a certain event or a certain task that may increase the activity in a related brain area is increased. Increased activity, however, implies increased synaptic transmission and more learning. Attention leads to increased activity, and activity is a prerequisite for learning.

These arguments are very similar to Neisser's (1976) arguments concerning the anticipatory cycle, almost 25 years ago. He showed how attention primes the system for activity by reducing the number of possible actions. Furthermore it strengthens the development of control programs (or "schemata" in the words of Neisser).

Bach-y-Rita (in press) gave another argument for the important role of meaningfulness. He indicated that neuromodulators may play a role in changing the excitation threshold of neurons. Mood states (attention, emotion, motivation) influence these neuromodulators and by this the rate and efficacy of learning.

\section{PRINCIPLE OF EQUIVALENT SITUATIONS}

This principle has its roots in motor research at the beginning of the $1900 \mathrm{~s}$, particularly in the identical elements theory of Thorndike (1914). The theory stated that when the learning takes place in context $\mathrm{X}$ and the application will take place in context $Y$, then for an optimal transfer it is necessary that both contexts have as much as possible elements in common. Elements are the general characteristics of the task or performance context. Holding (1976), who up-dated the insights of Thorndike, argued that the amount and the direction of transfer is related to the similarity of the stimulus and response characteristics of the learning and application situation. Related to motor tasks (e.g., walking in a hospital vs. walking on a street) this means that the more similarities these two tasks have, the more transfer will take place. However, it is important to note that similarities are not limited to the motor skills only, also the characteristics of the learning environment are stored in memory during the learning process. When the learning or treatment context differs essentially from the application context, less transfer will take place. Hence, the similarity between skills plus the similarity between contexts determine the amount of transfer that will take place after learning. However, since research addressing the problem of learning transfer has been minimal during recent years, we should be modest in giving suggestions for solving the problem.

\section{CONCLUSIONS}

In this paper, we argued that the recent insight in neural plasticity and learning may play a role for improving treatment after damage of the neuromotor system. We stressed the crucial role of sensory input. Indeed, on the basis of the mentioned experimental results, it is clear that input forms a sine qua non for change and learning. We argued that manipulating the input may offer additional opportunities for spinal cord patients, patients with amputated limbs, and stroke patients. Furthermore, it was argued that concepts derived from research into motor learning may be used in neurological rehabilitation. We stressed the importance of variability and meaning and we emphasized the role of the context, the learning environment. The 
latter is important since many therapists tend to ignore this role and expect significant transfer of learning even when the therapeutic context differs essentially from the conditions outside the hospital. In fact, a learning landscape is needed that enables the therapist to exercise under conditions that are ecologically relevant, that is to say, under conditions that have many elements in common with the daily life situation. These learning landscapes should form part of any modern design of a rehabilitation center. Until now, only a few centers have appreciated these principles. This article contains also arguments against the employment of rigidly formalized treatment methods that ignore the individual character of the damage by assuming that all patients may be treated according to the same protocol. Hence, neuroscience, neuropsychology, and motor control theory have something to offer for neurological rehabilitation, even at the very concrete level.

\section{REFERENCES}

Allard T, Clark A, Jenkins WM, Merzenich. 1993. Reorganization of somatosensory area 3B representations in adult owl monkeys following digital syndactyly. J Neurophysiol 66: 1048-1058.

Aroniadou-Anderjaska, V, Keller A. 1995. LTP in the barrel cortex of adult rats. NeuroReport 6: 22972300.

Bach-y-Rita P. In press. Conceptual issues relevant to present and future neurological rehabilitation. In: Levin H, Grafman J, eds, Neuroplasticity and Reorganization of Function After Brain Injury. New York, NY, USA: Oxford University Press.

Bliss TV, Lømo, T. 1973. Long-lasting potentiation of synaptic transmission in the dentate area of the anaesthetized rabbit following stimulation of the perforant path. J Physiol 232: 331-356.

Brasil-Neto JP, Cohen LG, Pascual-Leone A, Jabir FK, Wall RT, Hallett M. 1992. Rapid reversible modulation of human motor outputs after transient deafferentation of the forearm: A study with transcranial magnet stimulation. Neurology 42: 1302 1306.
Brasil-Neto, JP, Valls-Sole J, Pascual-Leone A, Cammarota A, Amassian VA., Cracco R. 1993. Rapid modulation of human cortical motor outputs following ischaemic nerve block. Brain 116: 511-525.

Bruehlmeier M, Dietz V, Leenders KL, Roelcke U, Missimer J, Curt A. 1998. How doest the human brain deal with a spinal cord injury? Eur J Neurosci 10: 3918-3922.

Calford MB, Tweedale R. 1990. Interhemispheric transfer of plasticity in the cerebral cortex. Science 249: 805-807.

Cohen, LG, Weeks RA, Sadato N, Celnik P, Ishii K, Hallett M. 1999. Period of susceptibility for cross-modal plasticity in the blind. Ann Neurol 45: 451-460.

Corbetta M, Miezin FM, Dobmeyer S, Shulman G, Petersen SE. 1991. Selective and divided attention during visual discriminations of shape, color and speed: functional anatomy by positron emission tomography. J Neurosci 11: 2383-2402.

Dietz V, Wirz M, Curt A, Colombo G. 1998. Locomotor pattern in paraplegic patients: Training effects and recovery of spinal cord function. Spinal Cord 36: 380-390.

Dombovy ML. 1993. Rehabilitation and the course of recovery after stroke. In: Whisnant JP, ed, Stroke: Populations, Cohorts, and Clinical Trials. Oxford, UK: Butterworth-Heinemann; 218-238.

Churchland PS, Sejnowski TJ. 1992. The Computational Brain. Cambridge, Massachusetts, USA: MIT Press; 560.

Crair MC, Malenka RC. 1995. A critical period for long-term potentiation at thalamocortical synapses. Nature 375: 325-328.

Cruikshank SJ, Weinberger NM. 1996. Receptive field plasticity in the adult auditory cortex induced by Hebbian covariance. J Neurosci 16: 861-875.

Hall, EJ, Flament, D, Fraser, C, Lemon, RN. 1990. Non-invasive brain stimulation reveals reorganized cortical outputs in amputees. Neurosci Lett 116: 379-386.

Hirsch JA, Gilbert CD. 1993. Long-term changes in synaptic strength along specific intrinsic pathways in the cat visual cortex. J Physiol (London) 461: 247-262.

Hebb, DO. 1989. The Organization of Behavior. New York, NY, USA: Wiley; 335.

Hochstenbach J, Mulder Th, van Limbeek J, Donders R, Schoonderwaldt H. 1998. Cognitive decline 
following stroke: A comprehensive study of cognitive decline following stroke. J Clin Exp Neuropsychol 20: 503-517.

Holding DH. 1976. A approximate transfer surface. J Motor Behav 8: 1-9.

James, A. Stroke treatment trials yield disappointing results. Lancet 1997. 349: 1673.

Khaw, KT. Epidemiology of stroke. J Neurol Neurosurg Psychiatry, 1996. 61: 333-338.

Kwakkel G. 1998. Dynamics in Functional Recovery After Stroke. PhD thesis. Amsterdam, The Netherlands: Free University; 250.

LeDoux J. 1996. The Emotional Brain: The Mysterious Underpinnings of Emotional Life. New York, NY, USA: Touchstone; 384.

Lodder J. In press. Neuroprotection in stroke: Analysis of failure and alternative strategies. Neurosci Res Commun.

Magill RA. 1998. Motor Learning: Concepts and applications. Boston, Massachusetts, USA: McGraw-Hill; 326.

McGaugh JL, Introini-Collison IB, Cahill LF, Castellano C, Dalmaz C, Parent MB, Williams CL. 1993. Neuromodulatory systems and memory storage: Role of the amygdala. Behav Brain Res 58: 81-90.

Milner B, Corkin S, Teuber HL. 1968. Further analysis of the hippocampal amnesic syndrome: 14-year follow-up study of H.M. Neuropsychologica 6: 215-234.

Mulder Th, Hochstenbach J. In press. Motor control and learning: Implications for neurological rehabilitation. In R. Greenwood et al., eds, Handbook of Neurological Rehabilitation. Hillsdale, California, USA: Erlbaum.

Mulder Th, Den Otter R, Van Engelen B. In press. The regulation of fine movements in patients with Charcot Marie Tooth, Type Ia: Some ideas about continuous adaptation. Motor Control.

Neisser U. 1976. Cognition and Reality: Principles and Implications of Cognitive Psychology. San Francisco, California, USA: Freeman; 230.
Nudo RJ, Wise BM, SiFuentes F, Milliken GW. 1996. Neural substrates for the effects of rehabilitative training on motor recovery after ischaemic infarct. Science 272: 1791-1794.

Ramachandran VS, Hirstein W. 1998. The perception of phantom limbs. Brain 121: 1603-1630.

Ramachandran VS, Blakeslee S. 1998. Phantoms in the Brain: Human Nature and the Architecture of the Mind. London, UK: Fourth Estate; 328.

Ritter H, Schulten K. 1986. On the stationary state of Kohonen's self-organizing sensory mapping. Biol Cybern 54: 99-106.

Rossini PM, Martino G, Narici L, Pasquarelli A, Peresson M, Pizella V, et al. 1994. Short-term brain "plasticity" in humans: transient finger representation changes in sensory cortex somatotopy following ischaemic anesthesia. Brain Res 642: 169-177.

Schmidt RA. 1988. Motor Control and Learning: A Behavioral Emphasis. Champaign, Illinois, USA: Human Kinetics; 689.

Singer W. 1986. The brain as a self-organizing system. Eur Arch Psychiatr Neurol Sci 236: 4-9.

Spitzer M. 1999. The mind within the net: Models of Learning, Thinking and Acting. Cambridge, Massachusetts, USA: MIT Press; 359.

Stefan K, Kunesch E, Cohen LG, Benecke R, Classen J. 2000. Induction of plasticity in the human cortex by paired associative stimulation. Brain 123: 572-584.

Wagenaar, RC. 1990. Functional Recovery After Stroke. $\mathrm{PhD}$ thesis. Free University of Amsterdam, the Netherlands: Free University Press; 229.

Warrington EK, Weiskrantz L. 1974. The effect of prior learning on subsequent retention in amnesic patients. Neuropsychologia 6: 169-177.

Wernig A, Nanassy A, Muller S. 1998. Maintenance of locomotor abilities following Laufband (treadmill) therapy in para- and tetraplegic persons: Followup studies. Spinal Cord 36: 744-749.

Whiting HTA. 1984. Human Motor Actions: Bernstein Reassessed. Amsterdam, the Netherlands: North Holland; 634. 

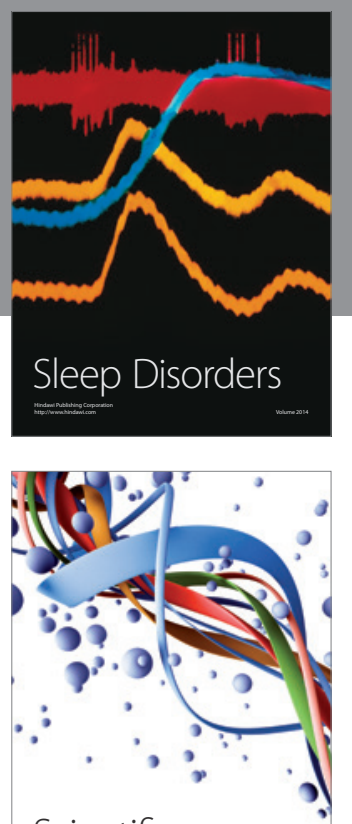

Scientifica
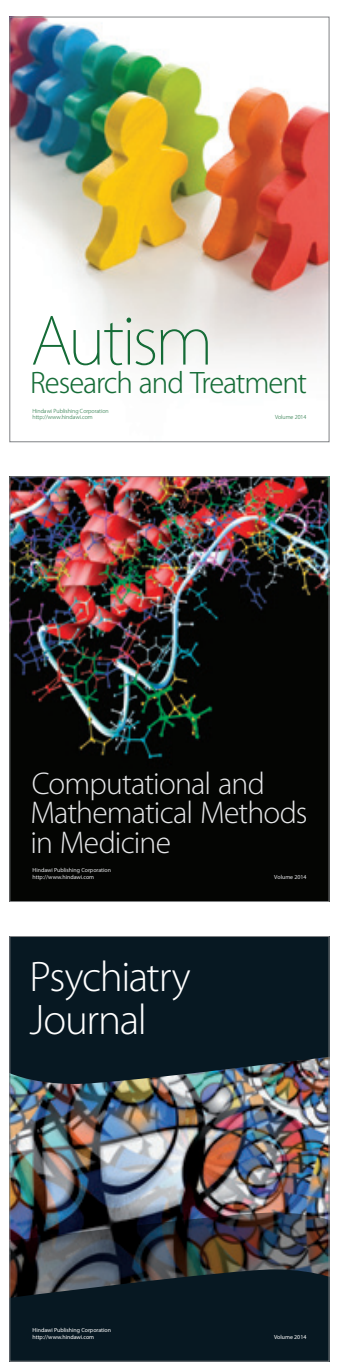
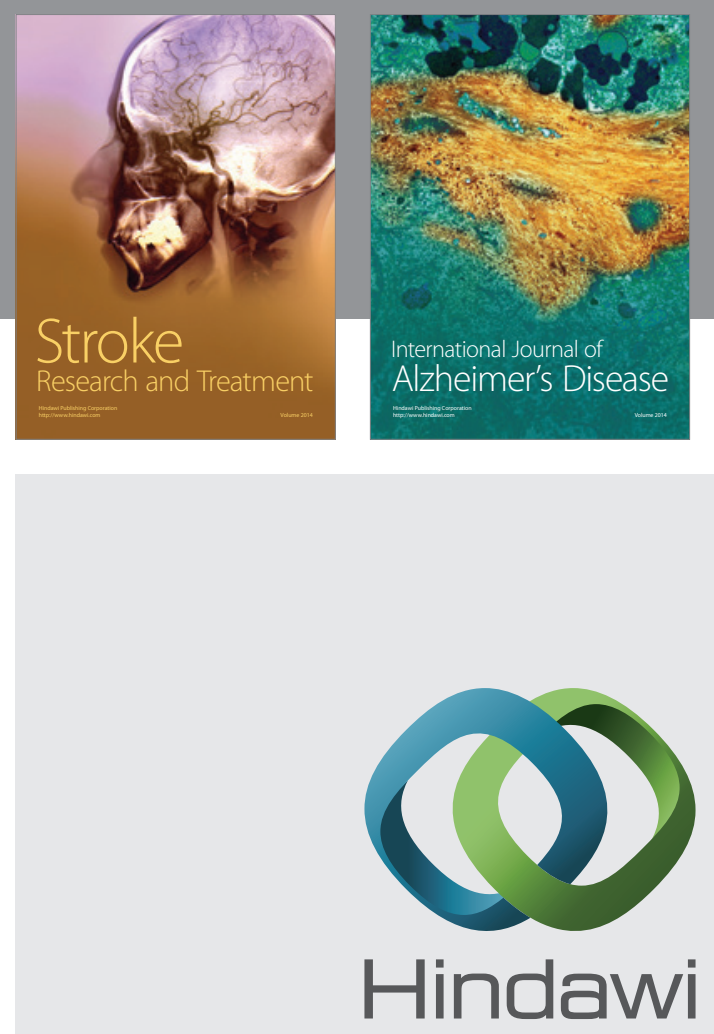

Submit your manuscripts at

http://www.hindawi.com
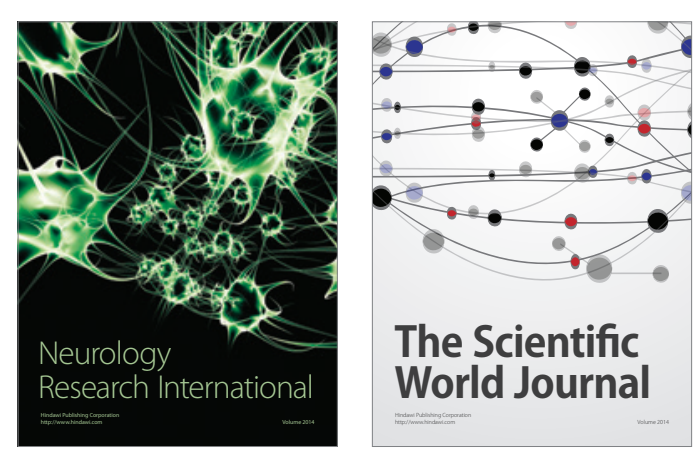

The Scientific World Journal

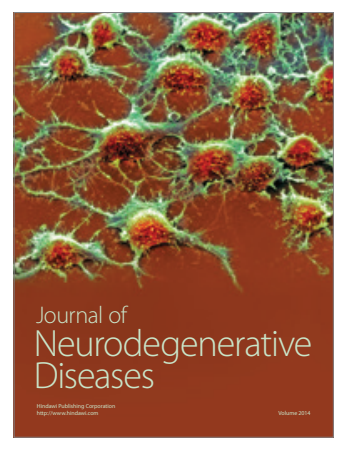

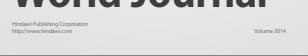

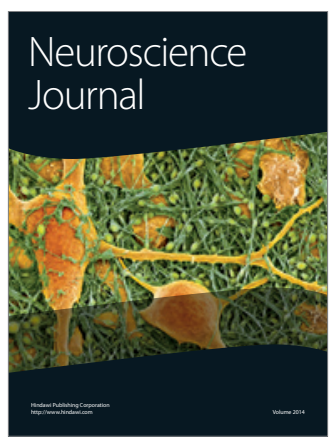

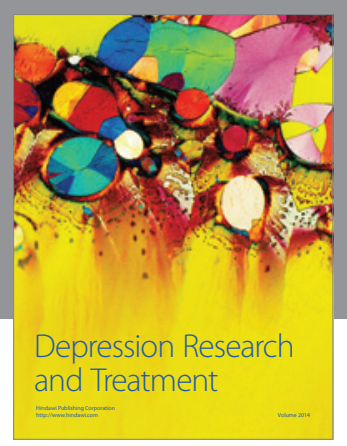
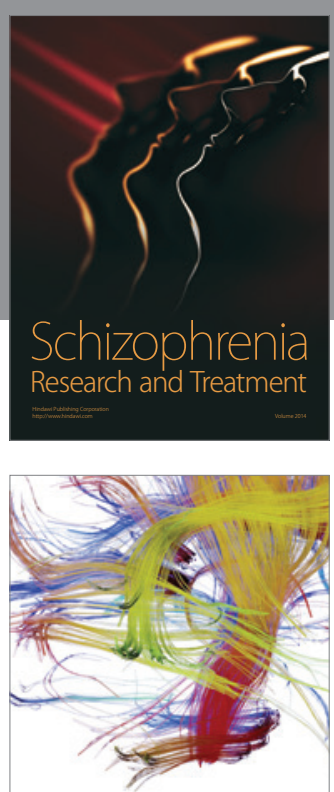

Brain Science

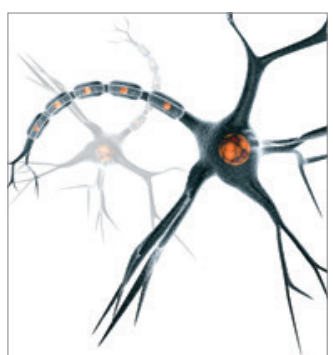

Neural Plasticity
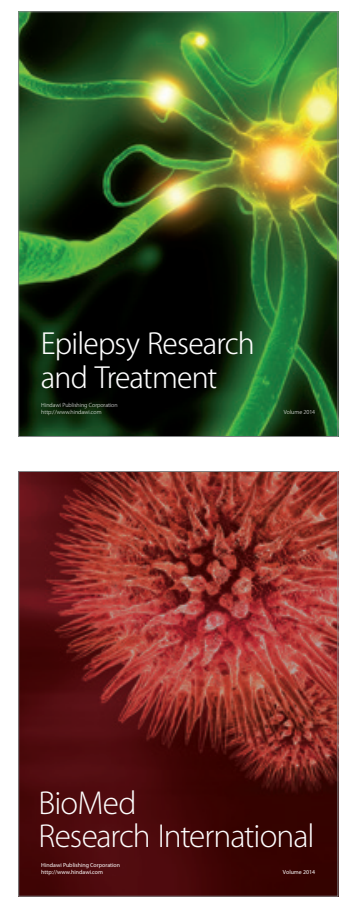

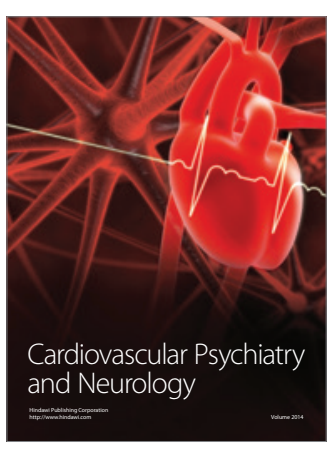

Parkinson's

Disease
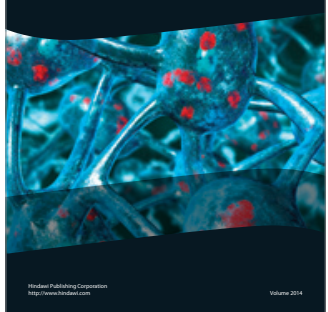